\title{
Forward Detectors in ATLAS: LUCID, ZDC and ALFA
}

\author{
L.Fabbri ${ }^{1}$ \\ on behalf of the ATLAS Collaboration and Forward Physics Working Group \\ 1- INFN and University of Bologna \\ Via Irnerio 46, 40128 Bologna - Italy
}

\begin{abstract}
In order to determine the experimental cross sections for the observed physics processes and point out signatures of new physics an estimation of the absolute luminosity is needed. ATLAS will make extensive usage of detectors in the forward region each one with a different task. LUCID, ZDC and ALFA will be able to monitor the bunchby-bunch luminosity, help the beam tuning and provide a measurement on both the absolute luminosity and the total cross section. The methodologies for luminosity monitoring and total cross section estimation at the LHC will be reviewed in this paper along with the dedicated detectors of the ATLAS experiment.
\end{abstract}

\section{Importance of Luminosity Determination}

The measurement of the cross section of any physics process is subject to the determination of the collected absolute luminosity. Just to illustrate an example we look at the Higgs boson. As reported in the ATLAS Physics TDR [2], the luminosity measurement may represent the main source of systematic for the determination of the Higgs branching ratios. This is well illustrated by Fig. 1, which shows the uncertainty on the production cross section of the Higgs particle times its decay branching ratio for various channels as a function of the Higgs mass. Two scenarios are reported depending on the uncertainty on the luminosity determination: $10 \%$ and $5 \%$, at an integrated luminosity of 300 $\mathrm{fb}^{-1}$. The Figure shows the error scales as the uncertainty on the luminosity, pointing out that this is the dominant contribution.

Also to observe deviations from the Standard Model (SM) the absolute luminosity play a leading role. In fact a careful study of well known processes will be one of the first steps of the LHC experiments as it can provide possible signatures of new physics which consist in deviations with respect to the SM predictions. To discriminate among standard processes and new physics a determination of the luminosity at the level of 2-3 $\%$ is required. To get to this goal different methods are needed for cross check and to minimise

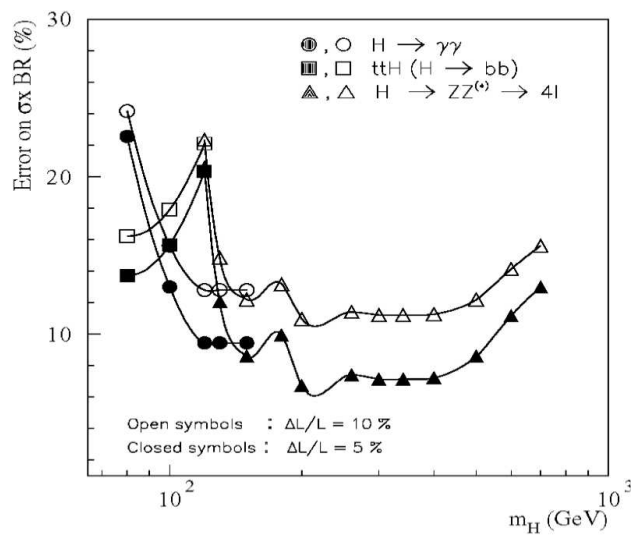

Figure 1: Uncertainty on the production cross section of the Higgs particle times its decay branching ratio for various channels as a function of the Higgs mass at an integrated luminosity of $300 \mathrm{fb}^{-1} \quad$ [2]. the systematic. The luminosity measurement will therefore be the result of a combined effort of different detector components, including trigger and data acquisition (DAQ) systems.

A constant luminosity monitoring during the acquisition is also essential to ensure good data quality and an efficient use of the available beam. For example the pre-scaling of 
the various trigger chains, running in parallel, must be constantly adjusted according to the beam intensity variations with time. Moreover the feedback from the experiments is a valuable tool for the machine operators to monitor the quality of the delivered beam and also, in case of danger, to react promptly. Quantities as the bunch-to-bunch relative luminosity, constantly updated, inform about the bunch structure and the beam background during the whole data tacking.

\section{Luminosity from Machine Parameters}

Luminosity can be determine from the machine parameters following the formula:

$$
L=F \frac{f \sum_{i=1}^{k_{b}} N_{1 i} N_{2 i}}{4 \pi \sigma_{x}^{*} \sigma_{y}^{*}}=F \frac{f k_{b} N^{2}}{4 \pi \epsilon_{N} \frac{\beta^{*}}{\gamma}}
$$

where $F$ is a factor which account for the beam crossing angle, $f=11.2 \mathrm{kHz}$ is the beamrevolution frequency, $N_{1 i}$ and $N_{2 i}$ are the numbers of protons in the colliding bunches, $k_{b}$ is the number of bunches and $\sigma_{x}^{*}$ and $\sigma_{y}^{*}$ are the transverse bunch widths at the interaction point. Assuming $N_{1 i} \simeq N_{2 i} \simeq N$ and expressing the beam dimensions via the normalized emittance $\epsilon_{N}$, the value at the impact point of the amplitude function $\beta^{*}$ and the beam Lorentz factor $\gamma$, it can be given by the right side of the Formula.

The most important effects that limite the accuracy of such determination are the evaluation of $\sigma_{x}^{*}$ and $\sigma_{y}^{*}$ at the impact point (IP) while they are measured far from that point, the extrapolation of the number of protons in the core of the bunch from the current measured by machinist and also other effects like beam-beam interaction (hourglass effect) and beam crossing angle determination [3]. All these effects make the maximum precision obtainable with this method of the order of $5-10 \%$. Moreover, the luminosity acquired by experiment is in general different from the one delivered and measured by accelerator, so a determination that takes into account the status of the whole experiment strictly connected to the experimental trigger (ATLAS live luminosity) is needed.

\section{The ATLAS Strategy}

The ATLAS goal is measure luminosity with 2-3\% of uncertainty. The strategy adopted in order to achieve such goal is a multi-step procedure which uses different techniques and different detectors fully described in [4]. The principal method to measure absolute luminosity uses the ALFA detector in Roman Pots. The aim is to measure the elastic scattering in the coulomb-nuclear interference region to come simultaneously to luminosity and total cross section determination. Due to the challenging technique the possibility to use the optical theorem is also taken into account as a back-up solution.

The principal detector to monitor luminosity and beam stability is LUCID, a particle counter using Cherenkov radiator. Once it has been calibrated LUCID will measure the absolute luminosity in all beam and luminosity conditions. The luminosity determination from the beam parameters provide a first calibration of LUCID at the level of $10 \%$. When installed, the ALFA detector will provide a fine calibration in special running conditions. Also the rate of precisely calculable physics processes will be used as a constant monitor of the calibration of the luminosity detector at an expected level of $5 \%$. 
To improve luminosity from machine the Zero Degree Calorimeter (ZDC) will be used particularly in runs dedicated to Van der Meer scan.

\subsection{The LUCID Detector}

The LUCID detector consists of two aluminium vessels - filled with $C_{4} F_{10}$ Cherenkov radiator gas at about 1.1 bar - placed on each side of the beam interaction point at about 17 meters. The vessels are mounted around the beam-pipe and are suspended to stainless steel support cones fixed to the TX1S monoblocks. LUCID will be able to monitor the collision-by-collision luminosity by detecting and counting the number of charged particles coming from the IP and is designed to be used at luminosity up to $410^{33} \mathrm{~cm}^{-2} \mathrm{~s}^{-1}$. Each vessel contain 20 aluminium

\begin{tabular}{|l|c|}
\hline \multicolumn{2}{|c|}{ Main parameters per module } \\
\hline$\eta$ coverage & $\pm[5.6,5.9]$ \\
\hline no. of tubes & 20 \\
\hline Material & Mechanically polished Al \\
\hline Gas & $C_{4} F_{10}$ \\
\hline Pressure & $1.1-1.5 \mathrm{bar}$ \\
\hline Cherenkov angle & $3^{\circ}$ \\
\hline$<$ no. reflections $>$ & 3 \\
\hline Cherenkov threshold & $e^{-}: 10 \mathrm{MeV} p: 2.8 \mathrm{GeV}$ \\
\hline Read-out & $16 \mathrm{PMT}+4$ fibres \\
\hline expected dose & $7 \mathrm{Mrad} / \mathrm{y} @ 10^{34} \mathrm{~cm}^{-2} \mathrm{~s}^{-1}$ \\
\hline
\end{tabular}
tubes about $1.5 \mathrm{~m}$ long pointing to the IP. The Cherenkov light emitted with almost 3 degree half angle at 1 bar in $C_{4} F_{10}$ is trapped in the tube making an average 3.2 reflections and is collected by photomultiplyers either directly coupled to the tubes or linked via optical fibres. In phase I sixteen tubes per vessel are read out by photomultipliers directly mounted at the end of the tubes, while four are read out by bundles of optical fibres ending in

Table 1: LUCID most relevant parameters. multi-anode PMTs located out of the high radiation area. In Table 1 are reported the most relevant parameters of the detector. LUCID extends the $\eta$ coverage of ATLAS in the range from $\pm[5.6,5.9]$. The time resolution of few $n s$ ensures the capability to separate subsequent bunch crossing (BC) and makes the detector suitable for the online monitoring of the bunch structure. Moreover LUCID has a read out stream independent of level 1 trigger and ATLAS DAQ, this feature makes it able to monitor the beam independently of the ATLAS live status.

The aim of LUCID is to measure and monitor the number of $p p$ interactions per BC $\left(\mu_{L U C I D}\right)$ related to luminosity $(L)$ by the formula:

$$
\mu_{L U C I D}=\sigma_{p p} \times \epsilon_{L U C I D} \times L
$$

where $\sigma_{p p}$ is the $p p$ cross section and $\epsilon_{L U C I D}$ is the detecting efficiency.

To do that different methods are studied and implemented in the LUCID read out board (LUMAT) with the main assumption that the number of collision at IP follows Poisson statistic.

Zero counting method: extracts the average number of interaction in a certain period of time (luminosity blocks) comparing the frequency of $\mathrm{BC}$ with no detected interaction with the Poisson zero probability. This measurement is done for each BC providing bunchby-bunch luminosity. Increasing luminosity the lack of $\mathrm{BC}$ with no interaction induces to enlarge the luminosity blocks. Coincidence of the two modules can be required in order to reduce background sensitivity like beam gas and beam halo.

Hit counting method: counts the numbers of tubes with signal above a threshold (hits).

DIS 2009 
This number, divided for the mean number of hits expected per single interaction - measured at very low luminosity when a multiple interaction is most unlikely - gives the number of interactions. This method works for each $\mathrm{BC}$ too and is useable at large luminosity.

Track counting method: evaluates the number of primaries from the LUCID tube occupancy thanks to its low value for single interaction $(O c c \sim 0.05)$ The method works well up to 30 interactions per $\mathrm{BC}$ and is implemented bunch-by-bunch .

Hit multiplicity method extracts the number of interaction per bunch crossing by comparing the detector response with that expected by superposition of single interaction Due to the huge amount of information needed this method is not implemented bunch-by-bunch.

To conclude LUCID is a detector able to count the number of interactions per BC by measuring the mean number of charged particles produced in the $p p$ collision. Thanks to its feature it's able to monitor luminosity bunch-by-bunch and after calibrated it can also provide absolute luminosity.

\subsection{The ZDC Detector}

The Zero Degree Calorimeter (ZDC) [5] is a detector sensitive to neutral particles produced both in $p p$ and in Heavy Ions collisions. It is located in the very forward region at $140 \mathrm{~m}$ on both sides of the IP and at about zero degree to the beam $(\eta>8.3)$ providing a further hermeticity to the ATLAS experiment in the forward direction. It is a system of calorimeters - one electromagnetic (EM) module and three hadronic (HAD) modules per side - placed in the neutral beam absorber (TAN) protecting the aperture of the beam-pipe from the flux of high energy neutral particles produced in the forward direction. The ZDC will be exposed to a high radiation dose (up to $18 \mathrm{Grad} / \mathrm{y}$ at luminosity of $10^{34} \mathrm{~cm}^{-2} \mathrm{~s}^{-1}$ ), for this reason stringent requirement both to the detector structure and the electronics location had been necessary. The EM modules consist of 11 tungsten plates for a total of about $29 \chi_{0}, 1 \mathrm{~mm}$ diameter quartz rods cross the plates parallel to the beam and are then bent to the vertical direction. A multi-anode photomoltiplicators (MAPMT) placed on top of the module readout the Cherenkov light produced by showers inside the module itself. The pattern of hit rods provides the transverse position of the incident particle and the intensity of the light is proportional to its energy. The HAD modules are similar to the EM ones with the exception that only one of them has position sensing rods and these are grouped four by four into the MAPMT. Three modules are still installed in each TAN located in section 1-2 and 8-1.

Both electromagnetic decaying neutral particles $\left(\pi_{0}, \eta\right.$ and $\left.K_{S}\right)$ and neutrons will be observed by ZDC, providing valuable measurements in a largely unknown kinematic region. The capability to measure both neutrons and $\pi_{0}$ will allow to observe also baryons as $\Lambda$ and $\Sigma$ and make ZDC an important instrument for forward physics studies.

As LUCID, ZDC will be used as luminosity monitor thanks to its capability to take measurement at the single bunch crossing level. Particularly it will be very helpful during the first day when the LHC parameters (crossing angle, IP position etc. ) will be tuned and during the runs dedicated to the Van der Meer scan.

\subsection{The ALFA Detector}

The ALFA detector complements ATLAS in the forward region (240 meters from the IP) and is aimed to measure the $p p$ elastic scattering at very small angles (i.e. t-values) in order to determine, at the same time, the absolute luminosity and the total $p p$ cross section $\sigma_{\text {tot }}$. 
The aim will be reached by measuring the elastic rate at small t-values where the Coulomb and the Nuclear amplitude are still equal and can be written:

$$
\frac{d N_{e l}}{d t}=L \pi\left|f_{C}+f_{N}\right|^{2} \sim L \pi\left|-\frac{2 \alpha}{|t|}+\frac{\sigma_{t o t}}{4 \pi}(1+\rho) \exp -b\right| t|/ 2|^{2}
$$

where $\rho$ is the real-to-immaginary part ratio of nuclear amplitude and $b$ is the nuclear elastic scattering slope parameter. Fitting the spectrum both $\sigma_{t o t}$, the luminosity $L$ and the parameters $b$ and $\rho$ are obtained. At LHC energy the Coulomb-Nuclear Interference Region corresponds to a scattering angle of almost $3 \mu \mathrm{rad}$. Due to this limits such measurement will be performed in special beam optic conditions. The foreseen parallel-to-point optics from the IP to the detector ensures that all particles scattered at the same angle impact at the same point in the detector, so that the measurement of such point translates directly to a scattering angle measurement at the IP. Such conditions will be met with a normalized emittance $\epsilon_{N} \sim 1 \mu \mathrm{m} \cdot \mathrm{rad}$ and a betatron-function at the IP $\beta^{*} \sim 2600 \mathrm{~m}$. Moreover a low instantaneous luminosity $10^{27}-10^{28} \mathrm{~cm}^{-2} \mathrm{~S}^{-1}$ with few filled bunches is foreseen.

The detector is housed in Roman Pots (RP) located between the sixth and the seventh quadrupole on both side of the IP. Two RP units separated by 4 meters will be installed in each side, each containing independently moving top and bottom pots, inside which a tracking detector based on scintillating fibers are housed. The detector is radiation tollerant up to 100 Grey/y and has a resolution up to $30 \mu \mathrm{m}$ with a rate capability of $1 \mathrm{MHz}$. At luminosity of $10^{27} \mathrm{~cm}^{-2} \mathrm{~s}^{-1}$ a statistical precision on luminosity itself at the level of $1.5 \%$ can be obtained in $\sim 100$ hours of data taking. Simulations show that a total statistical plus systematics uncertainty below $3 \%$ on luminosity can be reached. Full details on the measurement procedure and simulation can be found in [6].

\section{Conclusions}

Three detectors dedicated to luminosity measurement are arranged by ATLAS in the forward region. A dedicated luminosity monitor (LUCID), situated at 17 meter from the IP on both side, ready for the first beam and able to monitor online bunch-by-bunch luminosity and provide absolute luminosity after calibration. ZDC, at 140 meters from the IP, will monitor the beam. Its aim is the study of heavy ions and pp physics but it will be useful to tune machine parameters in the early days. The ALFA detector, housed in roman pots at $240 \mathrm{~m}$, is dedicated to the measurement of total cross section. It will reach the Coulomb-Nuclear Interference Region and provide simultaneously total cross section and luminosity with an uncertainty of $2-3 \%$ in the whole LHC working range.

\section{References}

[1] Slides: http://indico . cern $\cdot$ ch/contributionDisplay $\cdot$ py? contribId=163\&sessionId=18\&conf Id=53294

[2] ATLAS Collaboration, The ATLAS Physics TDR (vol. 1) CERN-LHCC-99-15 1999.

[3] H. Burkhardt and P. Grafstrom, Absolute luminosity from machine parameters, LHC Project Report 10192007.

[4] ATLAS Collaboration, The ATLAS Experiment at the CERN Large Hadron Collider, J. Instrum. 3 S08003, 2008.

[5] ATLAS Collaboration, Zero Degree Calorimeters for ATLAS, CERN-LHCC-2007-001 I-0162007.

[6] H.Stenzel Luminosity calibration from elastic scattering ATL-LUM-PUB-2007-001, 2007. 